\title{
Compressive ultrasound can predict early pulmonary embolism onset in COVID patients
}

\author{
Davide Ippolito ${ }^{1,2} \cdot$ Carlo Capodaglio $^{1,2} \cdot$ Cesare Maino $^{1}{ }^{1} \cdot$ Rocco Corso $^{1,3} \cdot$ Davide Leni $^{1,3} \cdot$ Davide Fior $^{1,3}$. \\ Teresa Giandola $^{1,2} \cdot$ Maria Ragusi $^{1,2} \cdot$ Cammillo Talei Franzesi $^{1,2} \cdot$ Davide Gandola $^{1,2}$. Antonio Rovere ${ }^{1,3}$. \\ Sandro Sironi $\mathbf{i}^{2,4}$
}

Received: 30 June 2021 / Accepted: 12 September 2021 / Published online: 9 January 2022

(c) Società Italiana di Ultrasonologia in Medicina e Biologia (SIUMB) 2021

\begin{abstract}
Purpose To evaluate the usefulness of compressive ultrasound (CUS) for the diagnosis of deep vein thrombosis (DVT) in patients with SARS-CoV-2-related infection.

Methods 112 hospitalized patients with confirmed SARS-CoV-2 infection were retrospectively enrolled. CUS was performed within 2 days of admission and consisted in the assessment of the proximal and distal deep venous systems. Lack of compressibility, or direct identification of an endoluminal thrombus, were the criteria used for the diagnosis of DVT. Pulmonary embolism (PE) events were investigated at computed tomography pulmonary angiography (CTPA) within 5 days of followup. Logistic binary regression was computed to determine which clinical and radiological parameters were independently associated with PE onset.

Results Overall, the incidence of DVT in our cohort was about $43 \%$. The most common district involved was the left lower limb $(68.7 \%)$ in comparison with the right one (58.3\%) while the upper limbs were less frequently involved $(4.2 \%$ the right one and $2.1 \%$ the left one, respectively). On both sides, the distal tract of the popliteal vein was the most common involved (50\% right side and $45.8 \%$ left side). The presence of DVT in the distal tract of the right popliteal vein (OR $=2.44495 \%$ CIs $1.084-16.624, p=0.038)$, in the distal tract of the left popliteal vein $(\mathrm{OR}=4.20195 \% \mathrm{CIs} 1.484-11.885, p=0.007)$, and $\mathrm{D}$-dimer values ( $\mathrm{OR}=2.12295 \% \mathrm{CIs} 1.030-5.495, p=0.003$ ) were independently associated with the onset on PE within 5 days.

Conclusions CUS should be considered a useful tool to discriminate which category of patients can develop PE within 5 days from admission.
\end{abstract}

Keywords Infections · Coronavirus · Tomography, X-Ray computed · Diagnostic imaging · Ultrasonography · Thrombosis · Embolism and thrombosis

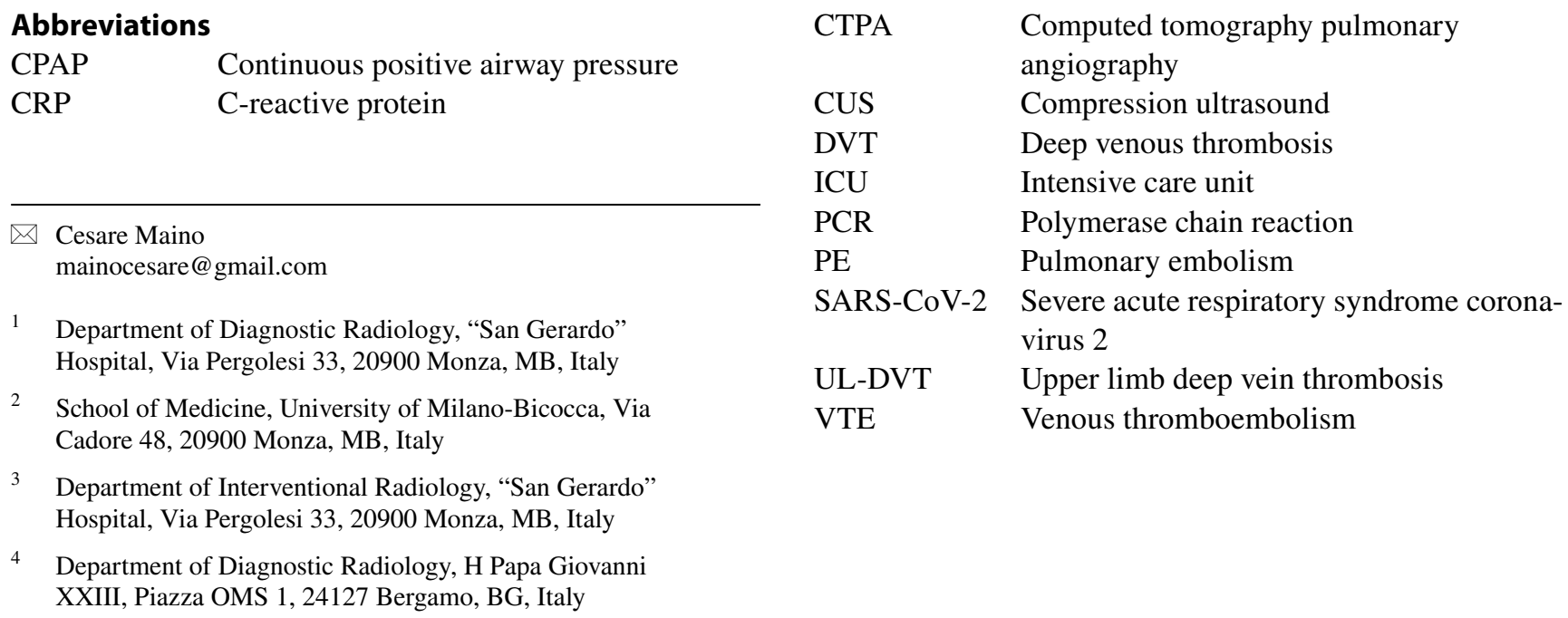




\section{Introduction}

When the pandemic caused by the SARS-CoV-2 virus spread through Europe in the first months of 2020, Italy was among the first European countries to register a surge in contagion and mortality, mainly in the northern areas. At the peak of the pandemic extension, Lombardy was the most affected region due to its high population density and the elevated elderly population [1].

During the first phase of the pandemic, there has been increasing awareness that venous thromboembolism (VTE) might contribute to acute respiratory failure in COVID19 patients and affect the clinical outcome. Moreover, coagulopathy has since been recognized as a fundamental complication in patients infected by SARS-CoV-2, in particular, those in critical condition or admitted to the ICU $[2,3]$.

Therefore, the need for thromboprophylaxis in these patients has been warranted, especially in the intensive care unit (ICU) setting. However, some groups suggest intensified doses based on the clinical or biological severity of SARS-CoV-2 infection as opposed to the standard low-molecular-weight dosing reported by most guidelines $[4,5]$.

Recent retrospective studies have found a significant prevalence of VTE, which often leads to pulmonary embolism (PE) thus contributing to a high mortality rate [2-6]. However, the performance of repeated computed tomography pulmonary angiography (CTPA) may not be sustainable in the context of a pandemic, where emergency departments and ICUs are frequently overwhelmed [7]. Numerous studies in recent literature have reported the routine use of compression ultrasound (CUS) as a useful tool to perform systematic screening investigation for the presence of deep venous thrombosis (DVT) in critical and non-critical SARS-CoV-2 patients with variable results, ranging in the incidence of $12 \%$ in non-ICU patients to $69 \%$ in severe ICU-setting even under anticoagulation $[8,9]$. Early detection of acute thromboembolic events in critical patients might therefore lead to a prompt medical or interventional treatment before PE occurs.

DVT commonly occurs in the lower extremities and is associated with PE. Viral infection in itself, prolonged bed rest, and the use of therapeutic devices such as those that might be implemented in ICU settings (i.e. invasive ventilation, central venous lines) have been recognized as predisposing factors for thrombosis. For patients with COVID-19, the possible correlation between the upper limb deep vein thrombosis (UL-DVT) and the application of continuous positive airway pressure (CPAP) has been described [10].
Our study aims to report the potential usefulness of CUS as a screening test for the diagnosis of DVT in hospitalized patients with SARS-CoV-2-related infection during the so-called "first wave" of the pandemic.

\section{Methods and materials}

The study was performed under the Declaration of Helsinki; clinical and radiological data were anonymized during data collection. Given the retrospective nature of the study and the presence of technical difficulties during the pandemic, informed consent will be waived, following article 89 of the GDPR EU Regulation 2016/679.

\section{Patients population}

All patients diagnosed with SARS-CoV-2-related infection, admitted from March 1st and May 31st 2020 were included in our study.

Inclusion criteria were: (1) age $>18$ years old, (2) conventional polymerase chain reaction (PCR) assayconfirmed diagnosis of SARS-CoV-2 infection, (3) laboratory test, including D-dimer value, blood cell differential, C-reactive protein (CRP) values, arterial blood gases (ABG) test, and (4) venous CUS of upper and lower limbs performed within 2 days of admission.

Patients without a complete venous CUS examination were excluded.

Critically ill patients were defined as those patients who required mechanical-assisted invasive or non-invasive ventilation in the ICU setting and/or with hemodynamic instability or requiring Extra Corporeal Membrane Oxygenation (ECMO). Standard patients were defined as those with severe symptoms, such as dyspnea which required non-invasive oxygen therapy.

All patients were receiving a standard dosage of prophylactic anticoagulation treatment (Enoxaparin 4000-6000 UI/die), established at the admission.

\section{Image procedure and analysis}

Venous CUS of legs and arms was performed within 1-2 days of admission using a portable scanner (MyLab XPro30-Esaote, Italy) with the linear probe, and consisted in the assessment of the proximal and distal deep venous systems. The distal investigated vessels were the posterior tibial, fibular, gastrocnemius (internal and external), gemellary, and soleal veins. The proximal veins of the upper limbs, including axillary, humeral and brachial, were examined. 
All venous segments were examined in real-time B-mode and by using color doppler mode in transverse and longitudinal sections. Lack of compressibility, or direct identification of an endoluminal thrombus, were the criteria used for the diagnosis of DVT.

Ultrasounds were performed by three interventional radiologists with more than 10 years of experience in this technique, separately.

The occurrence of PE was registered by CTPA, within 5 days follow-up.

\section{Statistical analysis}

Continuous variables were expressed as mean \pm standard deviation (SD), and compared by using the $U$ Mann-Whitney test. Categorical variables were expressed as median and IQR values and compared by using the $\chi^{2}$ test or Friedman test, as appropriate. Correlations were computed with the Pearson or Spearman correlation coefficients, as appropriate. For categorical variables, Odds Ratio (OR) was computed by using crosstabs, and $95 \%$ CIs were reported.

Median values obtained in the univariable analysis were used to compute backward stepwise logistic binary regressions to determine which clinical and radiological parameters were independently associated with PE onset. B values were considered as ORs and 95\% CIs were reported, as well.
All tests were two-sided, and the $p$-value $\leq$ of 0.05 was considered statistically significant. All the statistical analyses were performed by using IBM SPSS 26.0 (SPSS Incorporated, Chicago, Illinois, USA).

\section{Results}

\section{Demographic, clinical, and laboratory data}

By following the protocol design, 38 patients were excluded due to the lack of venous CUS of upper $(n=16)$ and lower $(n=22)$ limbs.

Finally, a total of 112 patients were enrolled, the majority was male $(n=82,73.2 \%)$ with a mean age of 62 years $( \pm 12)$. Laboratory data of the entire cohort are summarized in Table 1.

Overall, 48 patients (42.8) were judged as DVT positive, the majority male $(M / F=35 / 13)$. D-Dimer and hemoglobin values were significantly higher and slightly lower in the two subgroups $(2576.1 \pm 2269.8$ vs $1787.1 \pm 2430.6 \mathrm{ng} / \mathrm{ml}$, and $10.2 \pm 1.5$ vs $10.9 \pm 1.8 \mathrm{~g} / \mathrm{dl}$, $p=0.004$, and $p=0.009$, respectively) (Table 1 ).

There were no significant differences between patients in ICU or not-ICU according to the presence of DVT

Table 1 Clinical and laboratory data of the entire cohort, of patients DVT positive and negative and of patients PE positive and negative

\begin{tabular}{|c|c|c|c|c|c|c|c|}
\hline & $\begin{array}{l}\text { All } \\
(n=112)\end{array}$ & $\begin{array}{l}\text { DVT negative } \\
(n=64)\end{array}$ & $\begin{array}{l}\text { DVT positive } \\
(n=48)\end{array}$ & $p$ value & $\begin{array}{l}\text { PE negative } \\
(n=92)\end{array}$ & $\begin{array}{l}\text { PE positive } \\
(n=20)\end{array}$ & $p$ value \\
\hline Age & $62 \pm 12$ & $60 \pm 12$ & $64 \pm 10$ & 0.173 & $61 \pm 11$ & $66 \pm 13$ & 0.014 \\
\hline Sex male & $82(73.2)$ & $47(73.4)$ & $35(72.9)$ & 0.559 & $70(76.1)$ & $12(60.0)$ & 0.167 \\
\hline DVT positive & - & - & - & - & $34(36.9)$ & $14(70.0)$ & 0.007 \\
\hline ICU & $76(67.8)$ & $47(73.4)$ & $29(60.4)$ & 0.144 & $67(72.8)$ & $9(45.0)$ & 0.015 \\
\hline \multicolumn{8}{|l|}{ Labs } \\
\hline $\mathrm{WBC}\left(\times 10^{3} / \mathrm{mm}^{3}\right)$ & $11.7 \pm 9.6$ & $12.9 \pm 12.2$ & $10.1 \pm 3.8$ & 0.310 & $12.1 \pm 10.3$ & $9.8 \pm 4.8$ & 0.260 \\
\hline Neutrophyles $\left(\times 10^{3} / \mathrm{mm}^{3}\right)$ & $8.8 \pm 6.3$ & $9.73 \pm 7.7$ & $7.7 \pm 4.1$ & 0.431 & $9.3 \pm 6.8$ & $6.8 \pm 3.8$ & 0.176 \\
\hline Lymphocytes $\left(\times 10^{3} / \mathrm{mm}^{3}\right)$ & $2.6 \pm 9.6$ & $3.7 \pm 13.2$ & $1.27 \pm 0.9$ & 0.821 & $2.9 \pm 10.8$ & $1.3 \pm 1.5$ & 0.624 \\
\hline $\operatorname{PLT}\left(\times 10^{3} / \mathrm{mm}^{3}\right)$ & $281.8 \pm 129.3$ & $282.9 \pm 121.0$ & $280.2 \pm 140.8$ & 0.878 & $280.8 \pm 127.4$ & $286.2 \pm 141.0$ & 0.997 \\
\hline $\mathrm{CRP}(\mathrm{mg} / \mathrm{L})$ & $9.3 \pm 10.3$ & $9.6 \pm 10.3$ & $8.9 \pm 10.4$ & 0.644 & $9.8 \pm 10.4$ & $7.3 \pm 9.8$ & 0.158 \\
\hline D-Dimer (ng/mL) & $2110.6 \pm 2386.4$ & $1787.1 \pm 2430.6$ & $2576.1 \pm 2269.8$ & 0.004 & $2002.8 \pm 2422.4$ & $2601.2 \pm 2212.6$ & $\mathbf{0 . 0 3 0}$ \\
\hline $\mathrm{HB}(\mathrm{g} / \mathrm{dL})$ & $10.6 \pm 1.7$ & $10.9 \pm 1.8$ & $10.2 \pm 1.5$ & 0.009 & $10.6 \pm 1.7$ & $10.6 \pm 1.6$ & 0.619 \\
\hline ATIII (\%) & $192 \pm 533$ & $108 \pm 75$ & $287 \pm 778$ & 0.455 & $219 \pm 590$ & $77 \pm 16$ & 0.107 \\
\hline $\mathrm{a}-\mathrm{pH}$ & $7.42 \pm 0.1$ & $7.42 \pm 0.6$ & $7.43 \pm 0.6$ & 0.433 & $7.43 \pm 0.6$ & $7.43 \pm 0.5$ & 0.784 \\
\hline $\mathrm{a}-\mathrm{pO}_{2}(\mathrm{mmHg})$ & $95.5 \pm 24.1$ & $95.6 \pm 26.8$ & $93.8 \pm 20.0$ & 0.941 & $95.7 \pm 24.9$ & $94.1 \pm 20.1$ & 0.709 \\
\hline a-pCO $2(\mathrm{mmHg})$ & $45.6 \pm 9.6$ & $44.7 \pm 8.6$ & $46.8 \pm 10.9$ & 0.584 & $45.6 \pm 9.6$ & $45.6 \pm 9.9$ & 0.842 \\
\hline $\mathrm{a}-\mathrm{HCO}_{3}{ }^{-}(\mathrm{mEq} / \mathrm{L})$ & $30.0 \pm 5.1$ & $29.8 \pm 4.8$ & $30.3 \pm 5.5$ & 0.715 & $30.1 \pm 5.5$ & $29.6 \pm 5.3$ & 0.696 \\
\hline $\mathrm{a}-\mathrm{FiO}_{2}(\%)$ & $51.7 \pm 19.1$ & $49.0 \pm 18.1$ & $55.8 \pm 20.0$ & 0.130 & $52.3 \pm 19.2$ & $48.8 \pm 18.7$ & 0.634 \\
\hline $\mathrm{a}-\mathrm{SaO}_{2}(\% ; \mathrm{IQR})$ & $96(96-98)$ & $96(95-97)$ & $96(95-98)$ & 0.953 & 95 (94-97) & 96 (94-98) & 0.720 \\
\hline
\end{tabular}

$p$ values in bold represent statistically significant differences. "a-" = arterial blood gases test 
$(p=0.144)$. ICU patients were more frequently PE-negative in comparison to non-ICU ones $(p=0.015)$.

\section{Vascular districts involved by thrombosis}

The most common district involved was the left lower limb $(n=33,68.7)$ in comparison with the right one $(n=28$, 58.3). The upper limbs were less frequently involved $[n=2$ (4.2) right one and $n=1$ (2.1) left one, respectively].

On both sides the distal tract of popliteal vein was the most common involved portion $[n=24(50.0)$ on right side, $n=22$ (45.8) on left side] (Fig. 1A and 1B), followed by proximal tract of popliteal vein $[n=8$ (16.7) on right side, $n=8$ (16.7) on left side], and common femoral vein $[n=7$ (14.5) on right side, $n=4$ (8.3) on left side]. The superficial femoral vein was involved in five patients on the left side (10.4) and in seven patients on the right one (14.5).

Most patients showed two involved districts $(n=17$, $35.4)$, followed by one $(n=13,27.1)$, three $(n=3,6.2)$. Patients with at least four involved districts were 5 (10.4).

\section{DVT and PE}

During the following 5 days after CUS, a total of 20 patients (17.8) showed PE at CTPA, and only D-Dimer values were significantly higher in comparison with $\mathrm{PE}$ negative ones $(p=0.030)$ (Table 1).

Patients with DVT showed a higher risk to develop PE $(\mathrm{OR}=3.98,95 \% \mathrm{CIs}$ 1.39-11.32). The number of districts involved was significantly higher in patients with the onset of $\mathrm{PE}$ in the following 5 days $\left(\chi^{2}=16.96, p<0.0001\right)$.
Moreover, the number of districts involved showed a statistically significant correlation with the onset of PE $(r=0.655$, $p=0.005$ ).

By combining clinical and radiological data previously obtained, the logistic regressions showed that the presence of DVT in the distal tract of the right popliteal vein $(\mathrm{OR}=2.44495 \%$ CIs $1.084-16.624, p=0.038)$, in the distal tract of the left popliteal vein $(\mathrm{OR}=4.20195 \% \mathrm{CIs}$ $1.484-11.885, p=0.007)$, and $\mathrm{D}$-dimer values $(\mathrm{OR}=2.122$ 95\%CIs $1.030-5.495, p=0.003$ ) were independently associated with the onset on PE within 5 days. The ICU setting represented a protective factor regarding the onset of $\mathrm{PE}$ $(\mathrm{OR}=0.13695 \%$ CIs $0.064-0.289, p<0.0001)$. Results of logistic regressions are reported in Table 2.

\section{Discussion}

Venous thrombosis as a complication of viral infections is one of the prominent features in COVID-19 patients and often causes PE [11]. High D-dimer levels are common in COVID-19 pneumonia and are a marker for a worse prognosis. The nature of VTE in COVID-19 patients is complex, and treatment strategies regarding drug type and dosage continue to evolve. D-Dimer levels alone could not be sufficient as a reliable predictor for VTE, but rather a marker of poor outcome. Independent risk factors were not associated with a higher incidence of DVT at screening or PE; severity of the condition, either in ICU or in general wards was the principal association with VTE [5].

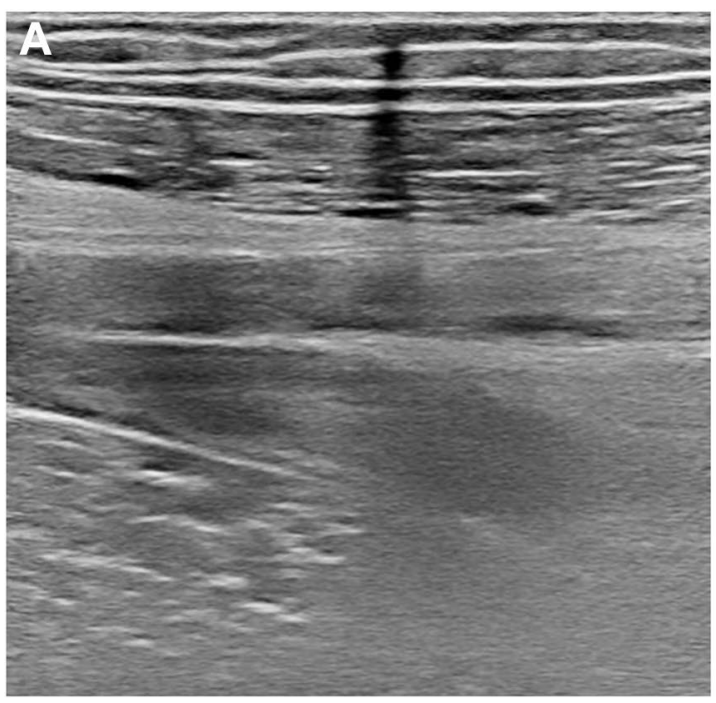

Fig. 1 A Grayscale ultrasound examination of common femoral vein demonstrating enlarged, non-compressible vessel, a typical example of DVT. B ColorDoppler examination of right deep and superficial

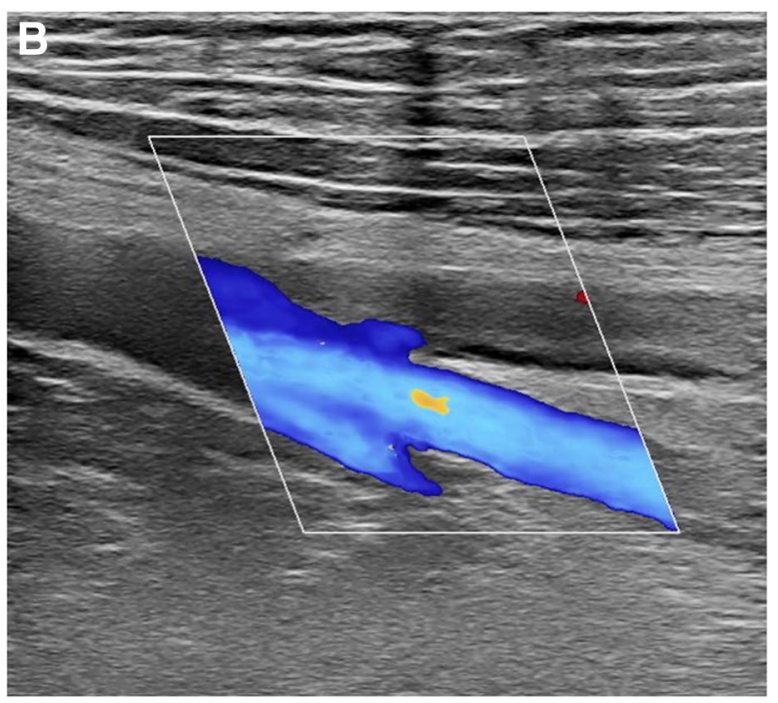

femoral veins showing absent flow in the superficial branch due to extensive thrombosis 
Table 2 Result of logistic regressions including clinical, laboratory and $\mathrm{ABG}$ data

\begin{tabular}{|c|c|c|c|}
\hline & Odds Ratio & $95 \%$ CIs & $p$ value \\
\hline Age & 1.245 & $0.469-2.032$ & 0.880 \\
\hline Sex male & 1.115 & $0.876-1.220$ & 0.766 \\
\hline ICU & 0.136 & $0.064-0.289$ & $<0.0001$ \\
\hline \multicolumn{4}{|l|}{ Deep Vein Thrombosis } \\
\hline Right common femoral vein & 0.026 & $0.000-2.577$ & 0.120 \\
\hline Right superficial femoral vein & 2.238 & $0.108-46.332$ & 0.602 \\
\hline Right popliteal vein (proximal tract) & 4.048 & $0.143-11.272$ & 0.412 \\
\hline Right popliteal vein (distal tract) & 2.444 & $1.084-16.624$ & $\mathbf{0 . 0 3 8}$ \\
\hline Left common femoral vein & 0.249 & $0.005-12.521$ & 0.487 \\
\hline Left superficial femoral vein & 2.531 & $0.372-11.305$ & 0.132 \\
\hline Left popliteal vein (proximal tract) & 0.311 & $0.010-9.521$ & 0.503 \\
\hline Left popliteal vein (distal tract) & 4.201 & $1.484-11.885$ & 0.007 \\
\hline \multicolumn{4}{|l|}{ Labs } \\
\hline $\mathrm{WBC}\left(\times 10^{3} / \mathrm{mm}^{3}\right)$ & 1.101 & $0.227-5.349$ & 0.905 \\
\hline Neutrophyles $\left(\times 10^{3} / \mathrm{mm}^{3}\right)$ & 2.981 & $0.481-18.460$ & 0.240 \\
\hline Lymphocytes $\left(\times 10^{3} / \mathrm{mm}^{3}\right)$ & 0.702 & $0.162-3.044$ & 0.636 \\
\hline $\operatorname{PLT}\left(\times 10^{3} / \mathrm{mm}^{3}\right)$ & 0.923 & $0.262-3.257$ & 0.901 \\
\hline CRP (mg/l) & 1.792 & $0.428-7.503$ & 0.424 \\
\hline D-Dimer (ng/mL) & 2.122 & $1.030-5.495$ & 0.003 \\
\hline HB (g/dL) & 0.760 & $0.230-2.514$ & 0.653 \\
\hline ATIII (\%) & 9.775 & $0.893-46.964$ & 0.062 \\
\hline $\mathrm{a}-\mathrm{pH}$ & 1.079 & $0.291-3.995$ & 0.909 \\
\hline $\mathrm{a}-\mathrm{pO}_{2}(\mathrm{mmHg})$ & 5.119 & $0.482-35.341$ & 0.175 \\
\hline a-pCO $(\mathrm{mmHg})$ & 1.448 & $0.236-8.902$ & 0.689 \\
\hline $\mathrm{a}-\mathrm{HCO}_{3}{ }^{-}(\mathrm{mEq} / \mathrm{L})$ & 3.464 & $0.632-18.990$ & 0.152 \\
\hline $\mathrm{FiO}_{2}(\%)$ & 0.408 & $0.091-1.832$ & 0.242 \\
\hline $\mathrm{a}-\mathrm{SaO}_{2}(\% ; \mathrm{IQR})$ & 0.233 & $0.024-2.306$ & 0.213 \\
\hline
\end{tabular}

$p$ values in bold represent statistically independent factors associated with PE
We evaluated both standard and intensive-care patients, all affected by SARS-CoV-2-related pneumonia, without subgrouping them according to the risk of PE, to avoid selection bias, showing that the overall incidence for DVT was about $43 \%$. All patients were undergoing standard prophylactic anticoagulation, and the difference in incidence between patients in ICU or not-ICU was not statistically significant $(p=0.144)$.

Our results are partially in line with the recent literature, which reports an incidence of venous thrombotic events in non-critical COVID patients of around 10\% [8-12], and incidence of DVT detected at the screening by CUS in ICU or critical patients ranging from 32 to $69 \%$ [9-13].

By analyzing our cohort, we found that the most common district involved was the lower limbs, more frequently the left in comparison with the right one ( $68 \%$ vs $58 \%$, respectively), while the upper ones were less frequently involved. In this setting, our results showed a more frequent involvement of the distal tract of the popliteal veins, in comparison with the proximal one (about 50\% vs 17\%, respectively).
On the other hand, by analyzing laboratory data, in our study we found out that high D-Dimer was the only biochemical parameter higher in patients with positive rather than negative DVT $(p=0.004)$ and patients with positive rather than negative PE $(p=0.003)$. Low hemoglobin levels were also associated with DVT-positive examinations $(p=0.009)$.

Combining clinical and laboratory data our study showed that patients with DVT showed a higher risk to develop PE $(\mathrm{OR}=3.98)$ in the following 5 days of hospitalization and that the number of districts involved by the DVT correlated with the onset of PE $(r=0.655, p=0.005)$.

Finally, by combining clinical and radiological data, the presence of venous thrombosis in the distal tract of both popliteal veins can be considered as independent factors towards the onset of $\mathrm{PE}(\mathrm{OR}=4.201$ and $p=0.007$ for right and $\mathrm{OR}=2.444$ and $p=0.038$ for left limb, respectively).

In these setting, we stressed the importance of CUS in patients affected with SARS-CoV-2: to the best of our knowledge, no guidelines provided a practical approach for the evaluation of DVT in both ICU and non-ICU patients. 
Therefore, as reported by Chen et al. [14], the mortality of patients DVT positive were higher in comparison with DVT negative, and this aspect should be carefully evaluated in all patients. Therefore, we think that CUS can be considered a useful screening tool for those patients hospitalized with a confirmed SARS-CoV-2 infection.

According to the Prospective Investigation of Pulmonary Embolism Diagnosis (PIOPED) II criteria [15], PE originates more frequently $(90 \%)$ in patients with DVT of the lower limbs. For this reason, we think that all hospitalized patients should be examined with CUS, to quick identify the presence of DVT and, consequently, taking a prompt decision regarding the best management available. As suggested by Parry [16], the confirmed diagnosis of DVT in patients suspected of PE may obviate the need for CTPA, especially considering those Countries with low healthcare resources or in overstretched hospitals.

Finally, the quick time-to-diagnosis, the low invasiveness, the possibility to be performed at bedside are some of important advantages of CUS, which can be ridden.

Our results and opinion are in line with Pieralli et al. [17], that suggested a surveillance protocol by serial CUS to identify patients with DVT, helping its detection and avoiding its possible consequences.

In the actual clinical practice, the indication for CTPA in patients affected with SARS-CoV-2 include an higher demand for oxygen, the presence of an acute respiratory distress syndrome (ARDS), increase in D-Dimer values, hemodynamic instability, or right heart chamber dilation [18-20].

Considering the aforementioned well-known advantages of CUS, we think that can be used as first-line imaging technique in all patients affected with SARS-CoV-2, to reduce radiation dose exposure and the risk of cross-infection due to transporting ill patients to the CT scanner room, to identify peripheral thrombi in the early phase of the disease and, finally, to stratify patients according to the risk of developing PE.

Pulmonary embolism detected by CTPA occurred within 5 days from screening in one-third of patients positive for DVT. Although this is a common occurrence in patients affected by venous thrombotic events, experience in performing CT in a large number of intensive care patients during the so-called "first wave" of the pandemic represented a huge burden for the radiology department and health care resources, especially given the need to adhere to strict infection control protocols.

Some limitations should be considered. Firstly, its retrospective nature. Secondly, given the state of emergency that our institution faced during that period and the strain put on the ICU, due to the high numbers of inpatients requiring intensive levels of care, even the general wards often temporarily supported critically ill or intubated patients: this might have created a sort of bias in assessing the severity of illness of enrolled patients.

To conclude, patients with COVID-19 pneumonia have a high incidence of DVT severe events, leading to an increased risk to develop PE during the hospitalization. As it is easily performable and repeatable, CUS should be considered as a fundamental tool in the initial management algorithm, both in ICU and in sub-critical settings, to determine the best management possible for each patient.

Funding No funding needed.

Availability of data and material All data generated or analyzed during this study are included in this published article.

\section{Declarations}

Conflict of interest Authors declared no conflict of interest.

Ethics approval Local Ethical Committee's review of the protocol deemed that formal approval was not required owing to the retrospective, observational, and anonymous nature of this study.

Consent to participate All patients signed the informed consent form to be eligible for this study.

Consent for publication All patients gave consent for information about themselves to be published in scientific journals.

\section{References}

1. Baccellieri D, Apruzzi L, Ardita V et al (2020) The "venous perspective" in Lombardia (Italy) during the first weeks of the COVID-19 epidemic. Phlebology 35:295-296. https://doi.org/10. 1177/0268355520925727

2. Klok FA, Kruip MJHA, van der Meer NJM et al (2020) Confirmation of the high cumulative incidence of thrombotic complications in critically ill ICU patients with COVID-19: an updated analysis. Thromb Res 191:148-150. https://doi.org/10.1016/j.thromres. 2020.04.041

3. Zhai Z, Li C, Chen Y et al (2020) Prevention and treatment of venous thromboembolism associated with coronavirus disease 2019 infection: a consensus statement before guidelines. Thromb Haemost 120:937-948. https://doi.org/10.1055/s-0040-1710019

4. Fontana P, Casini A, Robert-Ebadi H et al (2020) Venous thromboembolism in COVID-19: systematic review of reported risks and current guidelines. Swiss Med Wkly. https://doi.org/10.4414/ smw.2020.20301

5. Sakr Y, Giovini M, Leone M et al (2020) Pulmonary embolism in patients with coronavirus disease-2019 (COVID-19) pneumonia: a narrative review. Ann Intensive Care 10:124. https://doi.org/10. 1186/s13613-020-00741-0

6. Ippolito D, Giandola T, Maino C et al (2021) Acute pulmonary embolism in hospitalized patients with SARS-CoV-2-related pneumonia: multicentric experience from Italian endemic area. Radiol med 126:669-678. https://doi.org/10.1007/ s11547-020-01328-2 
7. Grasselli G, Pesenti A, Cecconi M (2020) Critical care utilization for the COVID-19 outbreak in Lombardy, Italy: early experience and forecast during an emergency response. JAMA 323:1545. https://doi.org/10.1001/jama.2020.4031

8. Santoliquido A, Porfidia A, Nesci A et al (2020) Incidence of deep vein thrombosis among non-ICU patients hospitalized for COVID-19 despite pharmacological thromboprophylaxis. J Thromb Haemost 18:2358-2363. https://doi.org/10.1111/jth. 14992

9. Llitjos J, Leclerc M, Chochois C et al (2020) High incidence of venous thromboembolic events in anticoagulated severe COVID19 patients. J Thromb Haemost 18:1743-1746. https://doi.org/10. $1111 /$ jth. 14869

10. Bozzani A, Arici V, Franciscone MM et al (2020) Severe acute respiratory syndrome coronavirus 2 infection and the upper limb deep vein thrombosis risk. Ann Vasc Surg 66:11-13. https://doi. org/10.1016/j.avsg.2020.04.037

11. Smilowitz NR, Subashchandran V, Yuriditsky E et al (2021) Thrombosis in hospitalized patients with viral respiratory infections versus COVID-19. Am Heart J 231:93-95. https://doi.org/ 10.1016/j.ahj.2020.10.075

12. Hamadé A, Jambert L, Tousch J et al (2020) Systematic duplex ultrasound screening in conventional units for COVID-19 patients with follow-up of 5 days. J Vasc Surg. https://doi.org/10.1016/j. jvsv.2020.11.019

13. Longchamp A, Longchamp J, Manzocchi-Besson S et al (2020) Venous thromboembolism in critically Ill patients with COVID19: results of a screening study for deep vein thrombosis. Res Pract Thromb Haemost 4:842-847. https://doi.org/10.1002/rth2. 12376

14. Chen S, Zhang D, Zheng T et al (2021) DVT incidence and risk factors in critically ill patients with COVID-19. J
Thromb Thrombolysis 51:33-39. https://doi.org/10.1007/ s11239-020-02181-w

15. Stein PD, Fowler SE, Goodman LR et al (2006) Multidetector computed tomography for acute pulmonary embolism. N Engl J Med 354:2317-2327. https://doi.org/10.1056/NEJMoa052367

16. Parry AH, Wani AH (2020) Pulmonary embolism in coronavirus disease-19 (COVID-19) and use of compression ultrasonography in its optimal management. Thromb Res 192:36. https://doi.org/ 10.1016/j.thromres.2020.05.022

17. Pieralli F, Pomero F, Giampieri M et al (2021) Incidence of deep vein thrombosis through an ultrasound surveillance protocol in patients with COVID-19 pneumonia in non-ICU setting: a multicenter prospective study. PLoS ONE 16:e0251966. https://doi. org/10.1371/journal.pone.0251966

18. Bompard F, Monnier H, Saab I et al (2020) Pulmonary embolism in patients with COVID-19 pneumonia. Eur Respir J 56:2001365. https://doi.org/10.1183/13993003.01365-2020

19. Jalaber C, Lapotre T, Morcet-Delattre T et al (2020) Chest CT in COVID-19 pneumonia: a review of current knowledge. Diagn Interv Imaging 101:431-437. https://doi.org/10.1016/j.diii.2020. 06.001

20. Safari S, Mehrani M, Yousefifard M (2020) Pulmonary thromboembolism as a potential cause of clinical deterioration in COVID19 patients; a commentary. Arch Acad Emerg Med 8:e52

Publisher's Note Springer Nature remains neutral with regard to jurisdictional claims in published maps and institutional affiliations. 